\title{
Pensionados para una ciencia en crisis: la JAE como mecenas de la anatomía macroscópica (1912-1931)
}

\author{
Raúl Velasco Morgado (*) \\ ${ }^{*}$ ) Área de Historia de la Ciencia (Historia de la Medicina). Universidad de Salamanca. \\ raulvmorgado@hotmail.com
}

Dynamis

[0211-9536] 2010; 30: 261-280
Fecha de recepción: 6 de octubre de 2008

Fecha de aceptación: 2 de junio de 2009

SUMARIO 1.-Introducción. 2.-La anatomía de la «Generación de Sabios» y la crisis de la anatomía macroscópica. 3.- La JAE y el estudio de las ciencias morfológicas: El nicho de la anatomía descriptiva macroscópica 4.-El período prebélico: Las primeras becas. 5. - Los pensionados en la Europa de la Gran Guerra. 5.1.-El tour de Joaquín Trías Pujol por los institutos anatómicos suizos durante 1917. 5.2.-Gumersindo Sánchez Guisande y la «anatomía dinámica». 6.—La descriptiva del tercer sistema: Los pensionados españoles en el laboratorio de Rouvière en el período de entreguerras. 7.-Conclusiones.

RESUMEN: Junto a la creación de instituciones investigadoras nacionales, la labor de mecenazgo de la Junta para Ampliación de Estudios (JAE) mediante becas (pensiones) y reconocimiento como pensionados de los científicos españoles del primer tercio del siglo XX fue la clave para llegar a la Edad de Plata de la ciencia española. En el caso de las ciencias morfológicas, la anatomía macroscópica era una ciencia casi cerrada y en crisis en favor de las ciencias microscópicas y la embriología. A pesar de este contexto, la JAE optó por promocionar esta ciencia, tanto para importar la pedagogía anatómica europea, como las técnicas y filosofía de la nueva «anatomía dinámica» que se gestaba en el continente. En este estudio analizamos a los pensionados en esta materia a través del archivo de la JAE y de los trabajos publicados por éstos. Concluimos que el aprovechamiento de éstas fue determinante para el intercambio internacional necesario para la reforma del paradigma una ciencia en «crisis», siendo la pedagogía y la técnica anatómicas las grandes protagonistas de esta renovación.

PALABRAS CLAVE: Anatomía, sistema linfático, miología, enseñanza universitaria, Siglo XX.

KEY WORDS: Anatomy, lymphatic system, miology, university teaching, 20th century. 


\section{A Francisco Efrén Pastor Jiménez,} nuestro maestro de Anatomía In memoriam

\section{Introducción}

A principios del siglo XX la anatomía macroscópica era una ciencia en declive, opacada por la brillante trayectoria de la morfología microscópica. Si bien es cierto que el paradigma descriptivo de aquélla estaba desfasado (no sólo por su potencialidad investigadora mínima, sino porque nuevas interpretaciones, más dinámicas, funcionales y aplicativas, se estaban imponiendo), no es menos cierto que seguía siendo un pilar fundamental en la formación médica, por lo que la pedagogía y técnica anatómicas seguían siendo muy necesarias entre los profesionales de la universidad de esta época.

Es éste, el primer tercio del siglo, un punto crítico para la anatomía macroscópica. En este momento la disciplina se encontraba a caballo entre la crisis de la macroscopía clásica y la introducción del nuevo modelo alemán. En este trabajo nos acercaremos, pues, a este período tomando como fuente primaria el archivo y las memorias de la que fue la institución más importante en cuanto al patronazgo de la ciencia española del período, la Junta para Ampliación de Estudios e Investigaciones científicas (JAE).

Tras ofrecer una breve perspectiva de la crisis a la que nos venimos refiriendo, haremos una revisión cuantitativa de las becas de la Junta destinadas a las ciencias morfológicas. Obtendremos así una visión de los intereses de los investigadores y el reflejo de la citada crisis en ellos. Ahondaremos, posteriormente, en cada una de las becas, siguiendo a los pensionados en sus viajes a través de Europa, estudiando el fenómeno de importación científica que se desprende de estos.

\section{La anatomía de la Generación de Sabios y la crisis de la anatomía macroscópica}

La nueva anatomía se venía gestando en Alemania durante la centuria decimonónica a medida que iba calando la concepción dinámica de especie a raíz de las teorías darwinistas. Este concepto iba unido al monopolio de 
las cátedras de anatomía alemanas por parte de zoólogos, en detrimento de los médicos. Estos se oponían a la nueva concepción en universidades como la de Heidelberg o Würzburg. No ocurría lo mismo en la cátedra de Oscar Hertwig en Jena, que recogía la tradición haeckeliana y que no encontró tantos obstáculos como en Heidelberg, precisamente la cuna de la escuela gegenbauriana, para la integración de la morfología comparada, la antropología evolucionista y la embriología ${ }^{1}$.

En el contexto del regeneracionismo krausopositivista, durante los años 80 del siglo XIX habían aparecido en la escena intelectual española un grupo de científicos a los que Laín bautizó como «Generación de Sabios»y que, con Santiago Ramón y Cajal a la cabeza, se constituyeron como profesionales plenamente europeos, integrados en la ciencia del continente, rematando el esfuerzo realizado en esta labor por parte de sus antecesores inmediatos, las llamadas «generaciones intermedias» ${ }^{2}$.

La anatomía de esa generación del 80 tiene al granadino Federico Olóriz Aguilera (1855-1912) como representante. Como tal, mantuvo una relación de amistad con Cajal y de admiración por el máximo exponente de la morfología de la etapa anterior: Julián Calleja Sánchez (1836-1913), con quien colaboró en su Compendio de Anatomía, precisamente en los apartados más dinámicos - los de anatomía comparada y embriología-. Él, por su parte, a pesar de contribuir con un manual de técnica anatómica y algunas observaciones sobre las suturas craneales y sobre la articulación témporo-mandibular, orientó sus investigaciones hacia la anatomía antropológica, fundamentalmente la biometría de cráneos y la dactiloscopia ${ }^{3}$. Sus coetáneos - Peregrín Casanova, López-Peláez y Villegas, entre otros-, consiguieron asentar una anatomía española que, a principios de siglo, integraba a la perfección la ontogenia y la filogenia en la explicación de la forma.

1. Sobre la evolución de la morfología alemana durante el siglo XIX véase Nyhart, Lynn K. Biology takes form: Animal morphology and the German Universities, 1800-1900. Chicago: University of Chicago Press; 1995.

2. López Piñero, José María. La aportación de las generaciones intermedias al saber Anatómico de la España del siglo XIX. Asclepio. 1971; 23: 95-130.

3. Un estudio sobre la crisis a la que nos referimos en este artículo y la figura del Prof. Olóriz la podemos encontrar en: García Carlos, Emilio. La crisis de la anatomía macroscópica en la obra de Federico Olóriz. Medicina e Historia, segunda época. 1974; 1-39. La biografía de Olóriz puede ser consultada en Guirao Pérez, Miguel; Guirao Piñeyro, Miguel. Federico Olóriz Aguilera. Biografía íntima del Profesor. Granada: Editorial Comares; 2008. 
Los anatomistas del primer tercio del siglo $\mathrm{XX}$ no hicieron otra cosa sino seguir la estela de esta Generación de Sabios y su «crisis macroscópica». La investigación de los niveles microscópicos estaba en pleno auge. Muy rotundo sobre la situación de la investigación morfológica se muestra Cajal en una carta enviada a Olóriz a finales de 1886 en respuesta a una consulta sobre conservación de piezas anatómicas:

«La Anatomía descriptiva está hecha, y para hallar un detalle nuevo hay que espigar porfiadamente durante muchos años: en tanto que la Histología normal, la Embriología, la Fisiología General nos brindan muchos problemas que resolver; el campo es vasto y tan poco cultivado que por muchos hombres se dediquen a su estudio, todavía quedarán muchos fenómenos en la sombra, y legaremos tarea a los venideros» ${ }^{4}$.

De este interés nace la archiconocida escuela histológica española que destacaría en la investigación biomédica básica en nuestro país durante décadas.

\section{La JAE y el estudio de las ciencias morfológicas: El nicho de la ana- tomía descriptiva macroscópica}

En este contexto crítico para la morfología, iniciado el siglo XX, las ciencias españolas viven lo que se ha venido denominando su «Edad de Plata» gracias a la creación de la JAE por parte del Ministerio de Instrucción pública. Esta institución, creada por R.O. en enero de 1907, tenía dos funciones fundamentales: establecer una red de centros de investigación nacionales, e internacionalizar la ciencia española a través de un sistema de pensiones en centros extranjeros ${ }^{5}$.

La investigación morfológica en los institutos propios de la JAE se centró en la histología, la morfología del sistema nervioso y la anatomía patológica microscópica. Se dedicaron a estas investigaciones el Laboratorio

4. Citado por García Carlos, n. 3, p. 14.

5. Barona, Josep L. Los laboratorios de la Junta para ampliación de estudios e investigaciones científicas (J.A.E.) y la Residencia de Estudiantes (1912-1939). Asclepio. 2007; 59 (2): 87-114 y Baratas Díaz, Luis Alfredo y Fernández Pérez, Joaquín. Becas de ampliación de estudios en Biología y Ciencias básicas de la Medicina en la España del primer tercio del siglo XX. Dynamis. 1993; 13: 247-263. 
de investigaciones biológicas (Instituto Cajal), el Laboratorio de histología normal y patológica de Pío del Río-Hortega, el Laboratorio de fisiología y anatomía de los centros nerviosos de Gonzalo Rodríguez Láfora, el Laboratorio de histología e histopatología del sistema nervioso del Museo González de Velasco (Museo Antropológico) y, en la Residencia de Estudiantes, el Laboratorio de anatomía microscópica de Luis Calandre. Como se puede apreciar, no existía ningún centro dedicado a la anatomía macroscópica, ni siquiera a una de sus variantes más dinámicas, la anatomía comparada. Para contar con un centro de estudio de la anatomía del desarrollo habría que esperar a 1936, cuando, en plena Guerra, se fundara el Laboratorio de embriología en Valencia.

Respecto a las pensiones para morfología, su estudio estadístico muestra las tendencias de la disciplina en este momento, en el que abundan los estudios de embriología (casi el 50\%), frente al 13\% que representa el estudio de la anatomía macroscópica descriptiva y su técnica y docencia, a cuyo nivel se pone la anatomía comparada. En total fueron seis los pensionados en anatomía descriptiva ${ }^{6}$. De ellos, sólo dos se dedicaron exclusivamente a este campo, mientras que los otros cuatro flirtearon con otras ramas de las ciencias morfológicas. Respecto a la categoría académica del pensionado, al igual que pasaba en otras áreas, estos fueron desde catedráticos de universidad, hasta jóvenes doctores -incluso uno de ellos es un estudiante de medicina interesado en la técnica anatómica- pasando por todas las categorías de profesores universitarios.

Por otra parte, existían dos tipos de pensionados: los que tenían una remuneración económica y los que gozaban de «condición de pensionado», que, en ocasiones, eran ayudados económicamente para los desplazamientos o les era conservado su sueldo si trabajaban para el Estado ${ }^{7}$. De los pensionados en anatomía, sólo dos recibieron pensión mensual, nombrándose a los otros cuatro como pensionados sin remuneración.

\footnotetext{
6. No se ha contabilizado aquí la beca concedida a Pedro Ara Sarriá por tres años para «estudios de anatomía en la República Argentina» en 1929, catedrático de la Universidad de Valencia por aquellos años, ya que no llegó a disfrutarse.

7. Formentín Ibáñez, Justo; Villegas, María José. Las pensiones de la JAE. In: Puig-Samper Mulero, Miguel Ángel, ed. Tiempos de investigación: JAE-CSIC, Cien años de investigación en España, Madrid: CSIC; 2007, p. 95-96.
} 


\section{El período prebélico: Las primeras becas}

El primero en ser pensionado por la JAE para estudiar técnica anatómica fue Sebastián Vizcaya Lozano, en $1912^{8}$. Pocos detalles nos proporciona su expediente. Sabemos que entonces tenía veintiséis años y que era interino en la cátedra de anatomía de Sevilla, donde fue alumno interno durante la carrera. Se había doctorado en 1910 con un trabajo sobre angiotecnia ${ }^{9}$. Sus investigaciones le habían permitido publicar «en diferentes revistas de Madrid, Barcelona, Sevilla y Alemania» y crear una técnica original para realizar preparaciones anatómicas, que había presentado en la exposición hispano-francesa de Zaragoza de $1908^{10}$. Ningún dato nos quedó de éste pensionado en los papeles de la JAE.

$\mathrm{Al}$ año siguiente se concedió consideración de pensionado al estudiante de medicina vasco de la Universidad de Valladolid José Salaverri Aranguren. El santurceño, que posteriormente dedicaría su quehacer a la dermatología ${ }^{11}$, solicitó la consideración mientras cursaba el segundo curso de la licenciatura "para estudios de anatomía e histología, especialmente para perfeccionarse en la técnica de obtención de preparaciones transparentes según el método del Dr. Spalteholz» ${ }^{12}$. Durante su estancia en el laboratorio de Werner Spalteholz (1861-1940) en Leipzig (Alemania) estudió su técnica para conseguir piezas anatómicas para el estudio de la angiología mediante la inyección de tintes en los vasos y el tratamiento del resto de tejidos para convertirlos en translúcidos. Resumió sus observaciones en un trabajo a su vuelta ${ }^{13}$, que nunca fue publicado.

8. Memoria correspondiente a los años 1912 y 1913. Madrid: Junta para Ampliación de Estudios; 1914, p. 162.

9. Vizcaya, Sebastián. Estudios de angiotecnia. Universidad Central; 1910.

10. Informe sobre la solicitud de pensión de Sebastián Vizcaya, 8 de marzo de 1912. Archivo de la JAE. Residencia de Estudiantes/152-364 (a partir de ahora, AJAE). Expediente de Sebastián Vizcaya y Lozano, f. 3.

11. Uruñuela Bernedo, Juan. Historia de la dermatología en el País Vasco. In: Calap Calatayud, Joaquín; Rodríguez Murillo, José Antonio, eds. Aspectos históricos de la Dermatología Médico-Quirúrgica y Venereología española. Barcelona: ISDIN; 1985, p. 201-205.

12. Salaverri Aranguren, José. Solicitud de consideración de pensionado, 10 de mayo de 1913. AJAE/13169, f. 1.

13. Salaverri Aranguren, José. Memoria sobre los ensayos del procedimiento del profesor Spalteholtz de Leipzig para conseguir la transparencia en piezas orgánicas macroscópicas. [Documento inédito]. Valladolid; 1913-1914. AJAE/131-69. 


\section{Los pensionados en la Europa de la Gran Guerra}

\subsection{El tour de Joaquín Trías Pujol por los institutos anatómicos suizos durante 1917}

Joaquín Trías Pujol (1888-1964), doctor en medicina y cirugía y licenciado en farmacia, cirujano militar nacido en Badalona, puede ser considerado como el anatomista-cirujano por excelencia de la España de principios del siglo XX. Este maridaje, que hunde sus raíces en la vocación práctica de la anatomía del siglo XVI, hace que estos profesionales sean relativamente ajenos a las tendencias microscópicas de la morfología y se sigan interesando por la macroscopía. Ésta es la razón de la existencia de cátedras, como la que consiguió Trías en la Universidad de Barcelona por concurso de traslado desde Zaragoza, con el apelativo de «anatomía topográfica y operaciones».

Su carrera académica había comenzado en 1916, cuando obtuvo la cátedra de técnica anatómica de la Universidad de Granada y solicitó una pensión a la JAE en concepto de «organización de los museos anatómicos y método de enseñanza de la anatomía». Especificaba en su petición un interés especial por «los modernos procedimientos de preparación de piezas macroscópicas, tanto normales como patológicas» ${ }^{14}$. Su anatomía, no obstante, se desliga de la anatomía clásica y se ve en cierta medida influido por las nuevas tendencias dinámicas. Así lo demuestra en la memoria presentada para las oposiciones a catedrático de anatomía descriptiva y embriología de la Universidad de Barcelona. Se trataba de una observación realizada durante una disección en la cátedra de Alejandro Planellas Llanos y llevaba por título Un caso de anomalía de la subclavia derecha y su explicación embriogénica ${ }^{15}$. Este trabajo ya se apartaba de la mera descripción macroscópica, para ahondar en la visión ontogénica de la variante anatómica.

\footnotetext{
14. Trías Pujol, Joaquín. Solicitud de pensión, 1 de marzo de 1915. AJAE/ 144-192. Expediente de Joaquín Trías Pujol, f. 1.

15. Original mecanografiado, con una fotografía de la disección macroscópica y dibujos esquemáticos de la hipótesis embriogénica, depositado en el AJAE.
} 
Lo que resulta innegable es la atracción que sentía el cirujano por la anatomía, asignatura por la que sentía - en sus propias palabras- «más vocación (...) que para el ejército» ${ }^{16}$. A pesar de ello, por avatares académicos diversos terminará cambiando la cátedra barcelonesa de anatomía por la de terapéutica quirúrgica en 1926.

Tras algunos inconvenientes, que trajeron aplazamientos para el disfrute de la beca, como las oposiciones a la cátedra de Granada o la burocracia de la licencia para el viaje que le comportaba su especial condición de militar, Trías comenzará durante el último trimestre de 1916 un recorrido por la totalidad de los institutos anatómicos de Suiza. La elección del país de destino estaba determinada por el contexto bélico internacional. A pesar de que existía en aquel momento entre los investigadores un gran interés por la anatomía alemana, la Junta, como es obvio, prefiere enviar a sus pensionados a los países neutrales ante la Gran Guerra ${ }^{17}$, lo que explica la abundancia de becas otorgadas para estudios en Suiza durante el bienio 1916-1917, y que disminuirá drásticamente tras la firma del Tratado de Versalles.

Pasó los meses de noviembre y diciembre de 1917 en el Instituto Anatómico de Ginebra junto con los profesores Auguste Eternod (18541932) y Sigismond Laskowski (1841-1928). A pesar de que se interesó por las técnicas de reconstrucción embriológica que estaba llevando a cabo Eternod, siguió con sus preferencias hacia la macroscopía. Allí encontró para ello un buen aliado con Laskowski, un profesor polaco exiliado a Suiza a finales del siglo XIX, autor de un reputado atlas y reconocido por la descripción en 1864 del método de la glicerina fénica para conservación de piezas anatómicas.

A finales de año se trasladó a Berna por tres meses. Allí asistió al curso de anatomía y disección del Anatomischen Institut dirigido por Hans Strasser (1852-1927), que completó con la asistencia a las conferencias semanales que impartía sobre embriología y anatomía comparada y a las autopsias y

16. Trías Pujol, Joaquín. Carta a Santiago Ramón y Cajal, 16 de marzo de 1915. AJAE/ 144-192. Expediente de Joaquín Trías Pujol. f. 2.

17. «La guerra europea ha continuado siendo un inconveniente, pero no un impedimento total para las pensiones en el extranjero. La Junta ha seguido enviando pensionados a los países neutrales, especialmente a Suiza, y aun a los beligerantes, cuando han estado alejados del teatro de la lucha, como los Estados Unidos, o cuando ha habido razones especiales para preferirlos, v. gr., en el caso de determinados estudios médicos». Memoria correspondiente a los años 1916 y 1917. Madrid: Junta para ampliación de estudios; 1918, p. XI. 
conferencias que se celebraban en el Instituto de Anatomía Patológica de Wegelim ${ }^{18}$.

Durante su estancia en el laboratorio de Strasser, además de proseguir con sus observaciones técnicas y pedagógicas, realizó una investigación sobre los linfáticos del endocardio ${ }^{19}$. Y es que, como veremos más adelante, la investigación de la distribución de los linfáticos constituía una de las pocas parcelas que quedaban aún abiertas en la vieja anatomía descriptiva macroscópica. El caso de los del endocardio encontraba una dificultad técnica para ser estudiados mediante inyección, puesto que al practicarla se repleccionaba, además, la vaina que recubría el fascículo atrio-ventricular y la red conductiva endocárdica descritas por His y Purkinje respectivamente. Recientemente, el japonés Sunao Tawara (1853-1952) había aceptado esta red como continuación del fascículo, y varios autores habían descrito la morfología de la red de Purkinje mediante inyección, pero nuestro autor intentó hacer una descripción diferencial con el sistema linfático utilizando las técnicas empleadas por el francés Renaut (1893) y, una más moderna (1909) del alemán E.J. Bartels para inyectar el sistema de Purkinje y los linfáticos, respectivamente. Describe así las diferencias fundamentales en forma, calibre y topografía entre los vasos linfáticos y la vaina inyectable que recubre el sistema de conducción del corazón. El trabajo fue publicado en su lengua materna en los Treballs de la Societat Catalana de Biologia 20 que dirigía su compañero del claustro barcelonés August Pi i Suñer. Aquí anunció que aparecería «un poco más ampliado, en una revista profesional de anatomía», publicación que no hemos podido localizar. No obstante, a pesar de que la repercusión de las descripciones no fuera grande, pues no conocemos ningún texto que le haga referencia, cumplía el objetivo de su estancia, familiarizándose con las modernas técnicas anatómicas europeas, en este caso la de la inyección de linfáticos, que en el momento no estaba muy difundida en España.

18. Memoria correspondiente a los años 1916 y 1917. Madrid: Junta para ampliación de estudios; 1918, p. 52.

19. Trías Pujol, Joaquín. La inyección de las redes de Purkinje y los linfáticos del endocardio. [Documento inédito]. Barcelona; 1917. Original mecanografiado conservado en el AJAE. Consta de cuatro figuras de las piezas anatómicas, tres de ellas fotografías y un dibujo en color.

20. Trías [Pujol], Joaquim. La injecció de les xarxes de Purkinje i dels limfàtics de l'endocardi. Treballs de la Societat de Biologia. 1917; 5: 191-202. 
Pero su obra más interesante es la observación de la docencia e investigación en los institutos anatómicos suizos, que resumió en una memoria bajo el título Organización de los institutos anatómicos y enseñanza de la anatomía en Suiza ${ }^{21}$. Terminó su ruta trasladándose a Zurich y Basilea durante las vacaciones de abril. Pasó quince días en Zurich, en el Instituto anatómico de George Ruge (1852-1919), discípulo de Gegenbaur, y en el Gehirn-anatomischen Institut (Instituto de neuroanatomía) que dirigía Constantin Von Monakow (1853-1930), donde coincidió con otro pensionado, José María Villaverde y Larrar, psiquiatra que realizaba investigaciones neuroanatómicas. Posteriormente, estaría otros ocho días en el Vesalianum de Basilea del estadounidense Hanson Kelly Corning (1861-1951), muy interesado en la anatomía dinámica y antropológica. Allí tuvo la ocasión de estudiar las colecciones embriológicas de His y de Kollman y, lo que a él más le interesaba, la colección de anatomía topográfica del actual director del instituto 22 .

La memoria de Trías se estructura en cinco partes: tras una introducción, una primera la dedica a la descripción general de los institutos de anatomía normal, una segunda sobre la infraestructura del de Berna, haciendo referencia a los demás visitados cuando tienen alguna diferencia notable que apuntar en este campo, una tercera sobre la finalidad de estas instituciones, una cuarta sobre la enseñanza práctica de la anatomía y una quinta dedicada a las técnicas anatómicas (conservación de piezas, induración para topotecnia, corrosión, método de transparencia de cortes de Spalteholz, estudio macroscópico del sistema nervioso central y técnica embriológica). Como anexo, añadió una breve reseña sobre la anatomía patológica suiza.

El pensionado encuentra en la Suiza alemana diferencias notables con la enseñanza de la anatomía en España. Critica, desde esta perspectiva, la excesiva especialización de los docentes de morfología. A diferencia de esto, en Suiza los profesores abarcan más materia, al menos en lo que a docencia se refiere. Mientras que en la Suiza francesa, como es obvio, se adoptó el modelo galo al igual que en España, y la histología iba unida a la

21. Trías Pujol, Joaquín. Organización de los Institutos anatómicos y de la enseñanza de la anatomía en Suiza. [Documento inédito]. Berna; 1917, 139 pp. Incluye 15 figuras en B/N. AJAE/144-192.

22. Trías Pujol, Joaquín. Memoria de actuación de pensionado, 15 de septiembre de 1917. AJAE/ 144-192. Expediente de Joaquín Trías Pujol. f. 20 b. 
embriología y la anatomía estaba aislada, en la Suiza germana, se adopta el modelo alemán o teórico, en el que la anatomía es una unidad: descriptiva y topográfica, en cuyo estudio, además, se incluyen la embriología, la histología e, incluso, la antropología, que son consideradas como ramas de aquella. Trías se muestra tajantemente a favor de esta última y decide importar a España la metodología docente de Strasser ${ }^{23}$, así como alguna de sus técnicas. De éstas le interesó su líquido de conservación de piezas ${ }^{24}$ que, siendo barato, «da a las preparaciones una claridad y resalte especial», a pesar de no conservar el color, que, en su opinión, es más útil en las piezas patológicas que en las normales.

En lo tocante a las instalaciones, destaca que se sorprende gratamente de la ubicación de la sala de disección de Zurich, en el segundo piso, con más luz y mejor potencial de ventilación y de la existencia de sala de estudio para alumnos en Berna, al modo de las del Reino Unido, con material bibliográfico y piezas anatómicas básicas. En relación a lo que ve, echa de menos en España la presencia de preparadores anatómicos, con una formación profesional específica, que facilitarían el trabajo de los investigadores. Algo que tampoco encontró en Suiza fueron escultores anatómicos profesionales, cuyas obras harían más fácil la docencia, como lo habían hecho en el siglo XIX. Por otra parte, la utilización de «cadáveres inyectados» en vez de «frescos», muy común en Suiza, estaba poco difundida en España, y a él le pareció la mejor manera de resolver «el obligado argumento o problema de la escasez de cadáveres».

En abril de 1917 Trías cree haber terminado su estudio de la anatomía suiza. Solicita entonces que los cuatro meses de pensión que no ha disfrutado le sean reservados hasta que termine la guerra y pueda desplazarse a Alemania para continuar sus trabajos ${ }^{25}$. Lamentablemente, esto no pudo llevarse a cabo por la organización burocrática de las becas, que sólo podían ser disfrutadas dentro de un mismo año. Establecido en su cátedra de Barcelona en 1918, no volvería a solicitar pensión a la JAE, lo que nos privó de su visión particular de la anatomía alemana de

23. El trabajo incluye, además de los planos del instituto de Berna, explicación y figuras de las mesas de disección y de disección-demostración modelo Strasser, sistema de almacenamiento de cadáveres, algunas muestras de piezas anatómicas elaboradas por el método de corrosión y una reconstrucción embriológica del sistema nervioso central de un reptil.

24. Líquido de Strasser: Agua de Lluvia: 1.000 cc; Formaline: 40; Sal común: 20.

25. Trías Pujol, Joaquín. Carta a Gonzalo Jiménez de la Espada, 1 de abril de 1917. AJAE/ 144-192. Expediente de Joaquín Trías Pujol, f. 14. 
principios del siglo pasado. Años más tarde, en 1930, se le concedería la condición de pensionado a Rafael Alcalá Santaella para que viajara al Instituto Anatómico de Munich y conociera «el plan y método de enseñanza de la anatomía» ${ }^{26}$ en este país. Por circunstancias académicas, Alcalá Santaella sólo pudo disfrutar de un mes de pensión, lo que no le permitió terminar los proyectos de investigación comenzados en Munich, ni proporcionarnos una visión tan precisa como la que Trías nos dejó de los institutos suizos.

\subsection{Gumersindo Sánchez Guisande y la «anatomía dinámica»}

En 1918, Gumersindo Sánchez Guisande (1894-1976) 27, entonces profesor auxiliar en la Universidad de Santiago, solicitó a la JAE continuar y terminar en Portugal con la condición de pensionado las investigaciones anatómicas que tenía comenzadas. Eligió para ello el Instituto anatómico de Oporto que dirigía Luis de Freitas Viegas (1869-1928) y el de la Universidad de Lisboa, de Henrique de Vilhena (1879-1958). Junto a este último realizó un trabajo de investigación para discernir si los músculos extensor común de los dedos del pie (m. extensor digitorum longus) y el peroneo anterior (m. peronaeus tertius) son en el hombre dos estructuras anatómicas diferentes. Comienza por un resumen de lo que los anatomistas clásicos habían escrito acerca de estas dos estructuras. Consultó para ello anatomistas dieciochescos alemanes como Albinus o Sömmerring, anatomistas europeos del «ochocientos» como Bichat, Cloquet o Cruveilhier ${ }^{28}$, a sus colegas contemporáneos Testut, Paterson, Chiarugi, Frohse y Fränkel, Dujarier y Merckel, y a los españoles de la generación anterior Batllés i Bertran de Luis y el omnipresente Calleja. Utiliza a continuación un arma poderosa para hacer este tipo de diferenciaciones: la anatomía comparada. Aunque había sido incluida por Calleja en su tratado, esta rama de la anatomía, como ya

26. Alcalá Santaella, Rafael. Oficio dirigido al Presidente de la JAE, 2 de agosto de 1930. AJAE/ 3-131. Expediente de Rafael Alcalá Santaella, f. 17.

27. La biografía de este futuro brillante catedrático de Anatomía puede consultarse en López Casamayor, José Luis. Gumersindo Sánchez Guisande: maestro de enseñanza y de vida. Almonacid de la Sierra: Ayuntamiento de Almonacid de la Sierra; 2007.

28. Cita también al cirujano británico John Neville Colley Davies-Colley (1842-1900), a los franceses Portal, Sappey, Fort, Teyle, Duchenne y a los alemanes Krause y Teyle (cuyas obras cita por la edición francesa). 
hemos apuntado, no se había difundido entre la mayoría de las cátedras de anatomía españolas, que no hicieron muchas aportaciones originales. Sánchez Guisande, por el contrario, la había incluido en su tesis doctoral (1916), que había realizado sobre los ligamentos interóseo y de Weitbrecht en el hombre y en los animales ${ }^{29}$, entrando en este mundo de la «anatomía dinámica» (filo y ontogénica). Sin embargo, en su trabajo como pensionado, no nos proporciona observaciones propias sobre anatomía comparada, sino un vasto saber libresco ${ }^{30}$. Para terminar, realizó una descriptiva de sus hallazgos en la disección de treinta y ocho cadáveres, desde los dos a los ochenta años de edad. Llegó a la conclusión de que en el hombre, ambos músculos son, al contrario de lo que la anatomía clásica mantenía, «una única individualidad muscular». Basó sus observaciones en que el peroneo anterior (que él describe como «haz metatarsiano del extensor común de los dedos del pie») no tiene homologías en la escala evolutiva. El trabajo fue publicado en la revista del instituto: Arquivo de Anatomía e Antropología ${ }^{31}$. El hallazgo, sin embargo, trascendió poco en la comunidad científica, quizás debido a que no publicó un compendio de osteomiología que hiciera que sus descripciones perduraran. Por todo ello, el músculo peroneo anterior sigue apareciendo en los principales tratados anatómicos como entidad individual sin que nadie haya dedicado una investigación en contra de lo postulado por Sánchez Guisande.

Su anatomía gegenbauriana, presente con anterioridad a la pensión, y afianzada por el contacto con la escuela anatómica portuguesa, marcaría su docencia e investigación durante su posterior trayectoria académica como catedrático de anatomía en las Universidades de Sevilla (1923-1928) y Zaragoza (1928-1936) y como director del Instituto Anatómico de Buenos Aires, tras exiliarse a consecuencia de la Guerra Civil. Sánchez Guisande logró inculcar así, tanto el nuevo concepto dinámico como el gusto por el estudio del sistema locomotor en sus discípulos, entre los que destacaría Luís Jiménez González (n. 1912).

29. Sánchez Guisande, Gumersindo. Investigaciones sobre los ligamentos interóseo y de Weitbrecht. Universidad Central de Madrid; 1916.

30. Entre las obras que consulta están las de los clásicos del siglo XVIII y XIX Meckel, Cuvier y Reinke, además de las obras de Le Doublé, Vogt y Yung, Chaveu, Arloing y Lesbre, Ellenberger y Baum, Sperino, Gratiolet y Allix, Hepburn y Roleston.

31. Sánchez Guisande, Gumersindo. El músculo extensor común de los dedos del pié del hombre. Arquivo de Anatomía e Antropología. 1919; 5: 1-52. 


\section{La descriptiva del tercer sistema: los pensionados españoles en el laboratorio de Rouvière en el período de entreguerras}

Como ya apuntábamos más arriba, si había un territorio por explorar en la cuasi-cerrada ciencia anatómica descriptiva macroscópica era el sistema linfático. Si tuviéramos que citar un nombre entre los anatomistas del siglo XX que dedicaron su investigación a esta materia tendríamos que mencionar al profesor de la Universidad de París Henri Rouvière (1876-1952). Dedicó su vida a la investigación de los linfáticos prosiguiendo la labor que había realizado Marie Philibert Constant Sappey (1810-1896). Sus investigaciones se materializaron en una completa monografía que apareció en 1932 en Francia bajo el título Anatomie des lymphatiques de l'Homme ${ }^{32}$ y que, aunque sí hay una versión inglesa, nunca se tradujo al castellano. Además, anteriormente, ya había publicado su celebérrimo tratado de anatomía, en el que demostraba su defensa de la anatomía dinámica, en la que se integra la embriología, la anatomía comparada y la fisiología. Siendo así un centro puntero en la investigación anatómica, y con esta perspectiva de la nueva morfología, no resulta extraño que no uno, sino dos de nuestros pensionados solicitaran estancias en el laboratorio de Rouvière.

El primero fue Rafael Alcalá Santaella (1896-1959), quien venía realizando unos modestos estudios morfológicos en el laboratorio de Calandre de la Residencia de Estudiantes. El cordobés, iniciando su etapa de posgrado, solicitaba en 1921 la condición de pensionado para «completar el estudio de la anatomía, en especial del sistema linfático, estudiando la técnica seguida por Sapey (sic)» ${ }^{33}$. Ésta consistía simplemente en la inyección de mercurio, como venía siendo clásico durante el siglo XIX, eligiendo correctamente el tipo de cadáver y la estación para hacerlo. La causa de la muerte debía ser una enfermedad crónica (utilizando sólo los cadáveres de niños para el estudio de cabeza, lengua, paladar blando y escroto), y tenía que ser practicada en verano, para facilitar la progresión del mercurio ${ }^{34}$. No ha llegado hasta nosotros ningún trabajo sobre los linfáticos realizado durante su es-

\footnotetext{
32. Rouvière, Henri. Anatomie des lymphatiques de l'homme. París: Masson; 1932.

33. Alcalá Santaella, Rafael. Solicitud de consideración de pensionado, 5 de abril de 1921. AJAE/ 3-131. Expediente de Rafael Alcalá Santaella. f. 1.

34. Olry, Regis; Motomiya, Kaoru. Paolo Mascagni, Ernest Alexandra Lauth and Marie Philibert Constant Sappey on the dissection and injection of the lymphatics. J Int Soc Plastination. 1997; 12 (2): 6-7.
} 
tancia, que duró un año completo. Imaginamos que se encontraba ocupado trabajando en su tesis de doctorado, que defendería al año siguiente en la Universidad Central sobre anatomía vascular del sistema renal ${ }^{35}$.

El interés despertado en Alcalá por los estudios embriológicos que en aquel instituto se gestaban hizo que optara por regresar años más tarde, solicitando de nuevo pensión a la JAE en 1934, siendo ya catedrático de anatomía descriptiva de Cádiz, para estudiar esta área pujante de la ciencia junto con Rouvière y el histólogo y embriólogo Christian Champy (18851962) ${ }^{36}$. No abandonaría, sin embargo, la macroscopía, especializándose en la anatomía del sistema renal, que evolucionó como complemento a su dedicación clínica a la urología ${ }^{37}$.

El segundo de los pensionados en el laboratorio de Rouvière, Francisco Orts Llorca (1905-1993), no pasó inadvertido por el instituto parisino. Su colaboración con el francés fue tal, que no tuvo más remedio que brindarle un agradecimiento en el prólogo de su monografía sobre el sistema linfático, junto a otros quince colaboradores ${ }^{38}$, ninguno procedente de España.

En 1931, Orts Llorca, por entonces profesor auxiliar de anatomía descriptiva de la cátedra de Ramón López Prieto en Valladolid, solicitó una pensión para estudiar «Sistema linfático y embriología general». Le fue concedida una pensión de doce meses de duración, que después se amplió, con destino a Francia y Austria. El primero de octubre de aquel año ${ }^{39}$ se encontraba ya en el Instituto de Anatomía parisino. Se trataba de una institución bien conocida por él, ya que dos años antes, recién terminada la licenciatura de medicina y cirugía, se había trasladado a la capital francesa para romper

35. Alcalá Santaella, Rafael. Anatomía macro y microscópica de las arterias renales. Universidad Central de Madrid; 1923. La tesis fue publicada aquel año en la imprenta madrileña de Javier Morata, y ampliada con sus investigaciones posteriores, años más tarde: Alcalá Santaella, Rafael. Estudio anatómico de los vasos y conductos excretores del riñón. Madrid: Javier Morata; 1929. Asimismo ganó el premio del curso 1935-1936 de investigación de la Real Academia de Medicina con el título «Vascularización e inervación del uréter humano».

36. Alcalá Santaella, Rafael. Solicitud de consideración de pensión, 28 de enero de 1934. AJAE/ 3-131. Expediente de Rafael Alcalá Santaella, f. 20.

37. Sobre su carrera urológica, véase Cánovas Ivorra, José Antonio; et al. Obra urológica y biografía del Profesor Rafael Alcalá Santaella (1896-1959). Análisis documental histórico. Archivo Español de Urología. 2004; 57 (8): 777-782

38. Rouvière, n. 32, p. III.

39. Memoria correspondiente a los cursos 131 y 1932. Madrid: Junta para ampliación de estudios e investigaciones científicas; 1933, p. 90. Lamentablemente no se conservan documentos relativos a esta primera pensión de Orts en el AJAE. 
su doble vocación - quirúrgica y morfológica- con una breve estancia compaginando el departamento de anatomía con el servicio de cirugía de Antonin Gosset. En el laboratorio de anatomía ya se había adiestrado en la técnica de inyección de linfáticos de Gerota modificada por Rouvière rectificando la concentración de turpentina y éter en el líquido a inyectar. Se había encargado de gran parte del estudio del sistema linfático ocular (glándula lacrimal, conjuntiva y párpados) para su monografía ${ }^{40}$, lo que se convirtió en la materia de su tesis doctoral. Ésta llevó por título Linfáticos de los órganos anexos del aparato de la visión, la terminó en el departamento de Valladolid y la presentó en la Universidad Central en 1931, obteniendo la calificación de sobresaliente ${ }^{41}$. En este lapso temporal, venía publicando trabajos sobre los linfáticos de otras áreas, como el año anterior, cuando había publicado un estudio sobre los de las paredes del abdomen ${ }^{42}$.

Concedida la pensión de la JAE aquel mismo año, le brindó la posibilidad de una estancia de dieciocho meses en el laboratorio parisino. El gran aprovechamiento de ésta se demuestra mediante numerosas publicaciones sobre el tercer sistema y dos de osteo-miología, además de comenzar a publicar algún estudio embriológico. Utilizó fundamentalmente como medio de difusión en Francia las publicaciones de la Sociedad Anatómica de París: Annales d'anatomie pathologique et d'anatomie normale médico-chirurgicale, y, en una ocasión la revista Comptes Rendus de l'Association des Anatomistes, usando el idioma del país vecino. Dos de ellos fueron traducidos al español y publicados en las revistas Policlínica de Valencia y Crónica Médica de Valencia, lo que expresaba la persistencia de su vinculación con la universidad donde cursó su licenciatura.

\footnotetext{
40. Gómez Santos, Marino. 5 grandes de la ciencia española: Severo Ochoa, Carlos Jiménez Díaz, Fernando de Castro, Francisco Grande Covián y Francisco Orts Llorca. Madrid: Biblioteca Nueva; 1968, p. 169.

41. Fue publicada en Crónica Médica en 1931 y, al año siguiente, en la Revista de Cirugía de Buenos Aires.

42. Orts Llorca, Francisco. Contribución al estudio de los linfáticos de la pared del abdomen. Crónica Médica de Valencia. 1930: 383-391.
} 
Durante el año 1931 publicó dos estudios en solitario sobre los linfáticos de la bóveda craneal ${ }^{43}$ y los del esternón, clavículas y vértebras ${ }^{44}$ respectivamente. En el año 1932 comenzó a publicar conjuntamente con investigadores franceses. Aquel año redactó junto con F. Vergé-Brian, quien venía investigando los linfáticos de los miembros desde unos años atrás, un trabajo sobre los linfáticos de los huesos largos ${ }^{45}$. Tres estudios publicados en 1932 y 1933 fueron el fruto de la colaboración con Julius Botár ${ }^{46}$, anatomista junto al que, además, comenzó a publicar trabajos embriológicos. En la misma línea que los anteriores y con la misma técnica de Gerota-Rouvière, describieron los linfáticos de la cadena simpática en el recién nacido y, lo que ha tenido una mayor repercusión clínica, los drenajes linfáticos de la próstata y su relación con los de la vejiga. Ayudaban así a sentar las bases anatómicas de la metástasis linfática del cáncer de próstata, completando, con la aplicación de la técnica de Gerota-Rouvière, lo establecido por Cuneo y Marcille entre 1901 y 1902. El experimento fue realizado con cincuenta piezas anatómicas de fetos, neonatos y niños, lo que aporta una visión dinámica ontogénica a esta estructura,

La repercusión de todo ello es que, en la monografía de Rouvière, Orts Llorca es citado de forma continua en el apartado de los linfáticos de la zona orbitaria. Describió por primera vez el «anillo linfático pericorneal», además de hacer otras aportaciones como el hallazgo de un pequeño ganglio «interruptor» a nivel del orificio externo del canal inguinal o un ganglio linfático a nivel de la séptima costilla, en el reborde inferior del pectoral mayor.

43. Orts Llorca, Francisco. Lymphatiques des os de la voute du crâne. Annales d'anatomie pathologique et d'anatomie normale médico-chirurgicale. 1931, (sin número ni paginación) [sabemos que fue reeditado en castellano con el título «Linfáticos de los huesos de la bóveda craneal» en Policlínica de Valencia el mismo año].

44. Orts Llorca, Francisco. Lymphatiques du sternum de la clavicula et des corpe vertebraux. Annales d'anatomie pathologique et d'anatomie normale médico-chirurgicale. 1931 (sin número ni paginación) [reed. en castellano en la Revista de Cirugía de Buenos Aires al año siguiente].

45. Orts Llorca, Francisco; Verge-Brian, F. Contribution a l'étude des lymphatiques des os longs des membres. Comptes Rendus de I'Association des Anatomistes, 21-23 mars 1932. 1932 (sin número ni paginación).

46. Orts Llorca, Francisco; Botar, J. Lymphatiques des ganglions de la chaine sympathique chez le nouveau-né. Annales d'anatomie pathologique et d'anatomie normale médico-chirurgicale. $9^{e}$ anée, 1932; 7 (s. págs.) ; Orts Llorca, Francisco; Botar, J. Collecteure lymphatiques de la prostate. Annales d'anatomie pathologique et d'anatomie normale médico-chirurgicale. 1933; 10 (1): 37-43 y Orts Llorca, Francisco; Botar, J. Vías linfáticas de la próstata y sus comunicaciones con la vegiga (sic). Crónica Médica de Valencia. 1933 (sin número): 37-43. 
Paralelo a estas investigaciones, Orts publicó dos trabajos sobre el sistema locomotor. El primero, en solitario, sobre el músculo manio ${ }^{47}$, y posteriormente otro más importante, junto a Rouvière, acerca de las intersecciones tendinosas de los músculos poligástricos ${ }^{48}$. En ellos se destallaban cómo la existencia de éstas se relacionaba con la poliinervación de estos músculos, evidenciando que los límites de inervación son perpendiculares a la dirección de las fibras musculares.

La anatomía de Orts, que se materializará a partir de mediados de siglo en uno de los textos más utilizados para la docencia de pregrado de la licenciatura de medicina, se verá influida - si bien con matices adquiridos durante sus posteriores estancias en Alemania- por la visión dinámica de Rouvière. Además, tal y como le pasó a Alcalá Santaella, los intereses macroscópicos de un cirujano-anatomista fueron opacados por la ciencia embriológica que se cultivaba en el laboratorio francés. La estancia de Orts en París se convirtió así en la primera experiencia de una brillante carrera como embriólogo que le dio reconocimiento internacional.

\section{Conclusiones}

Así pues, se puede concluir que, como continuación del proceso esbozado ya por las generaciones intermedias y afianzado por la «Generación de Sabios», la anatomía descriptiva macroscópica del primer tercio del siglo XX venía siendo desplazada por una anatomía «dinámica» que incluia la embriología y la anatomía comparada como armas más poderosas. Los pensionados de la JAE así lo demuestran en sus preferencias a la hora de solicitar los temas de investigación de sus estancias en el extranjero. La evolución a través de las dos décadas que separan la primera pensión concedida para el estudio de esta disciplina de la última resulta esclarecedora: mientras que los conceptos de las primeras becas se referían exclusivamente a la técnica anatómica, los científicos fueron progresivamente simultaneando sus estudios con las otras ramas, fundamentalmente la embriología, que

47. Orts Llorca, Francisco. ¿Existe el músculo manio? Crónica Médica de Valencia. 1932; (sin número): 740-748.

48. Rouvière, Henri Orts Llorca, Francisco. Les intersections tendineuses et les tendons intermédiaires des muscles polygastriques: leur signification. Boletín de la Universidad de Santiago, número extraordinario en homenaje al Prof. Rodríguez Cardaso. 1933 (s. págs.). 
terminaría por imponerse como objeto de investigación preferido durante las décadas posteriores.

Aunque aún está por hacer un estudio exhaustivo sobre el modelo de docencia de la anatomía española de principios del siglo XX, el estudio de la pensión de Joaquín Trías Pujol nos da una idea de la importancia que tuvo el contacto con los institutos anatómicos europeos en el proceso de importación de sus modelos por parte de los profesores pensionados. El catedrático catalán, sesgado por el contexto bélico que sólo le permitió realizar sus observaciones en Suiza, aboga por la implantación del modelo alemán de docencia de la anatomía, en la que la descriptiva siga ligada a la topográfica y también a la histología, la embriología y la antropología, que presupone ramas de la misma ciencia.

Por otro lado, nuestros pensionados importaron las últimas técnicas de investigación del sistema linfático, descubriendo uno de los escasos campos abiertos de la anatomía descriptiva, gracias a la técnica de inyección de Gerota. Tres de ellos, lo que corresponde a la mitad del grupo, hicieron algún tipo de investigación macroscópica del sistema linfático aprendiendo y aplicando dicha técnica.

Por último, se hace necesario anotar que, en el caso de la anatomía, sí que se cumplió uno de los grandes objetivos de la JAE: la internacionalización de la ciencia española. Aparecen así nombres de científicos españoles citados en uno de los tratados de anatomía de más calado internacional de la época, el de Rouvière. Éste cita a sus dos pensionados: Alcalá Santaella, en lo que a la anatomía macroscópica renal se refiere-sobre todo angiología- y Orts Llorca, respecto a los hallazgos linfáticos. 
Tabla 1. Pensiones de Anatomía (elaboración propia a partir de las memorias impresas de la JAE)

\begin{tabular}{|c|c|c|c|c|}
\hline Pensionado & $\begin{array}{l}\text { Año de } \\
\text { disfrute }\end{array}$ & Concepto & $\begin{array}{l}\text { País de } \\
\text { destino }\end{array}$ & $\begin{array}{c}\text { Área } \\
\text { Anatómica }\end{array}$ \\
\hline Sebastián Vizcaya Lozano & 1912 & Técnica Anatómica & $\begin{array}{l}\text { Francia y } \\
\text { Alemania }\end{array}$ & - Técnica anatómica \\
\hline José Salaverri Aranguren & 1913 & Anatomía e Histología & Alemania & - Técnica anatómica \\
\hline Joaquín Trías Pujol & 1916 & $\begin{array}{l}\text { Organización de los museos } \\
\text { anatómicos y método de en- } \\
\text { señanza de la Anatomía }\end{array}$ & Suiza & $\begin{array}{l}\text { - Pedagogía anatómica } \\
\text { - Técnica anatómica } \\
\text {-Anatomía topográfica } \\
\text {-Embriología }\end{array}$ \\
\hline Gumersindo Sánchez Guisande & 1918 & Investigación anatómica & Portugal & -Anatomía descriptiva \\
\hline \multirow[b]{2}{*}{ Rafael Alcalá Santaella } & 1922 & Estudios de anatomía & Francia & -Pedagogía anatómica \\
\hline & 1929 & $\begin{array}{l}\text { Organizaciones anatómicas } \\
\text { Anatomía y Embriología }\end{array}$ & Alemania & $\begin{array}{l}\text {-Anatomía descriptiva } \\
\text {-Embriología }\end{array}$ \\
\hline Francisco Orts Llorca & 1931 & Anatomía y Embriología & Francia & $\begin{array}{l}\text { - Anatomía descriptiva } \\
\text {-Embriología }\end{array}$ \\
\hline
\end{tabular}

